\title{
Identification et réappropriation des disciplines à travers la lecture - écriture de la série Harry POTTER
}

Nicole Biagioli, Solange Cartaut, Jean-Philippe Drouhard et JeanJacques Legendre

\section{(Q) OpenEdition}

\section{Journals}

Édition électronique

URL : http://journals.openedition.org/trema/799

DOI : $10.4000 /$ trema.799

ISSN : 2107-0997

Éditeur

Faculté d'Éducation de l'université de Montpellier

Édition imprimée

Date de publication : 1 octobre 2005

Pagination : 113-126

ISSN : 1167-315X

Référence électronique

Nicole Biagioli, Solange Cartaut, Jean-Philippe Drouhard et Jean-Jacques Legendre, « Identification et réappropriation des disciplines à travers la lecture - écriture de la série Harry POTTER », Tréma [En ligne], 24 | 2005, mis en ligne le 04 octobre 2010, consulté le 02 mai 2019. URL : http:// journals.openedition.org/trema/799; DOI : 10.4000/trema.799

Ce document a été généré automatiquement le 2 mai 2019.

Trema 


\title{
Identification et réappropriation des disciplines à travers la lecture - écriture de la série Harry POTTER
}

\author{
Nicole Biagioli, Solange Cartaut, Jean-Philippe Drouhard et Jean- \\ Jacques Legendre
}

\section{Introduction}

1 Pourquoi avons-nous choisi d'aborder les représentations scolaires, objet des interrogations de toutes les didactiques des disciplines, par le biais de la fiction? Les demandes directes sont peu fiables (effet bien connu des sociologues: les populations «fragiles» répondent selon l'image qu'elles veulent donner d'elles et pas selon leur pratique effective). La fiction à intertexte disciplinaire ou scolaire permet de prescrire le symptôme de confusion des discours et postures disciplinaires, comme les psychologues prescrivent le symptôme à leur patient pour l'aider à le maîtriser ${ }^{1}$. La projection sur les personnages romanesques agit pour les lecteurs à la fois comme une motivation à la réflexion méta - didactique, et une fois qu'elle est enclenchée, comme étayage.

Notre objectif était d'apporter des solutions aux problèmes professionnels induits par la coexistence des disciplines d'enseignement, qu'il s'agisse pour l'élève de se situer dans ses apprentissages ou pour l'enseignant de travailler en pluri - intervention. Ceci impliquait de démonter auparavant la pseudo-différenciation (découpage de surface des disciplines perçu de l'extérieur à travers la prescription (programmes, exercices) et l'évaluation (contrôles, concours). Celle - ci n'est pas dissipée et se voit même parfois accentuée par les projets interdisciplinaires (le projet met en retrait au profit de l'objectif commun certains des mécanismes de l'adaptation interdidactique, ou en assure une gestion empirique). La différenciation effective, elle, aboutit à un engagement situé de l'élève et l'aide à acquérir les identités multiples ${ }^{2}$ exigées par le système des disciplines. 
$3 \mathrm{Au}$ - delà des élèves du primaire et du collège, qui sont à la fois la cible explicite de la série de ROWLING et le public jugé le plus fragile face à la diversification des apports didactiques (seuil CM2 / 6e), la cible lectorale s'est avérée plus large ${ }^{3}$, englobant adolescents et adultes. Enseignants et parents jouent un rôle trop important dans le processus d'identification aux disciplines des élèves pour que leurs réactions soient ignorées, d'autant que leur intérêt pour la série laisse supposer une grande sensibilité aux problématiques scolaires.

Les procédures d'enquête et d'entretien s'imposaient pour identifier le contenu et les modalités de mobilisation des représentations dans les activités scolaires et dans la vie adulte. S'agissant d'une recherche appliquée comme on la pratique dans une ERTE (Equipe de Recherche en Technologie Educative), il fallait également susciter, observer, évaluer et s'il est nécessaire modifier, structurer ou renforcer les représentations, discours et pratiques inter didactiques. Pour cela, l'approche clinique des productions et des comportements individuels et la recherche - action impliquant des enseignants et leurs classes sont à la fois nécessaires et complémentaires.

Les pratiques imitatives constituant la base des processus d'identification, nous avons choisi comme objet de nos observations et de nos expérimentations des activités ludiques : théâtre, jeux de rôle ou jeux d'écriture. Enfin, pour ménager la transition entre les compétences acquises hors l'école et les compétences construites à l'école, nous avons abordé les représentations et les activités différenciatrices par la double entrée des pratiques ludiques privées et des pratiques ludiques didactisées par l'école.

\section{Enquête sur les représentations des disciplines et du système scolaire chez les lecteurs d'Harry POTTER}

6 Nous avons utilisé la saga Harry POTTER et plus spécialement sa description du système scolaire comme métaphore heuristique du fonctionnement de l'école actuelle. Les élèves ont été invités à comparer les situations romanesques avec leur propre expérience scolaire qui leur avait justement permis de se projeter dans la fiction et l'expérience des personnages. Le champ de référence est l'étude du personnage dans la fiction littéraire (1). Conformément à l'analyse de F. DE SINGLY (2), il a été décidé de réaliser une pré enquête par questionnaire. Associée à des entretiens, elle va permettre d'élaborer la problématique de l'enquête proprement dite à la lumière des réponses des acteurs sociaux.

7 Le sujet de l'enquête était la lecture experte d'Harry POTTER dans la composante particulière de la perception des rapports entre l'univers décrit dans la fiction et l'univers scolaire de des lecteurs. Des indicateurs (2), faits ou opinions, ont été élaborés pour pouvoir déterminer les comportements type associés à la lecture experte d'Harry POTTER considérée sous cet aspect. 
8 Les questions portaient sur les performances de lecture, l'intérêt de la fiction, la vie au sein de l'institution scolaire, les personnages et les matières enseignées.

Elles ont été posées à un échantillon spontané (2) de volontaires qui se sont cooptés. Ce choix a été motivé par le fait que le questionnaire concerne la lecture privée d'un ouvrage.

L'ensemble des questionnés (113) a lu entre trois et cinq tomes de Harry POTTER. $94 \%$ ont vu au moins 1 film et $49 \%$ ont joué à au moins un jeu vidéo. $8 \%$ ont lu un des livres prolongeant la saga.

11 L'attitude des sondés par rapport aux films fait apparaitre une nette préférence pour les livres. Les réponses aux questions concernant la vie à Poudlard révèlent des visions souvent opposées, en particulier en ce qui concerne la répartition des élèves en " maisons ». En effet, si 13 des sondés considèrent comme positive cette répartition qui doit selon eux favoriser une saine émulation, 20 sont d'un avis totalement opposé : ils considèrent que cette division induit des conflits qui nuisent au fonctionnement harmonieux de l'établissement. Au moins un des aspects de cette vie en internat est considéré comme positif par 53 sondés (vie en commun, relations étroites avec les professeurs) alors que seuls 5 d'entre eux y voient un aspect négatif (éloignement de la famille).

12 En ce qui concerne le personnage préféré des lecteurs, seule une faible minorité a porté son choix en dehors du triangle des protagonistes : Harry, Hermione, Ron. L'identification avec un personnage du même sexe n'est pas systématique. Les trois héros principaux ne sont pas appréciés de manière identique: si Hermione est clairement qualifiée d'intelligente ou de bonne élève, Harry est, lui, considéré simplement comme " le héros » sans qu'aucune qualité particulière ne lui soit systématiquement attribuée. Quant à Ron, il est qualifié majoritairement de «drôle ». Ainsi, et c'est peut être une raison de son succès, les qualités de Harry ne sont pas clairement affichées et ce flou le rend plus universel. En outre, il est étonnant que, même chez les adultes, voire les enseignants, le choix ne se soit porté sur un professeur que 8 fois ; ceci en dépit du rôle central joué par le corps enseignant dans les romans. Ceci est peut être attribuable à la qualité du roman qui incite le lecteur adulte à reprendre ses yeux d'enfant.

Les enseignants préférés des lecteurs sont DUMBLEDORE, MCGONAGALL, HAGRID et LUPIN. Les qualificatifs qui leur sont attribués sont : gentil, protecteur, juste, sévère, sage, à l'écoute, est ou essaye d'être bon professeur. L'avis des sondés vis - à - vis des professeurs semble, à de rares exceptions près, être indépendant de la matière enseignée et basé sur des critères pour lesquels la compétence n'est qu'accessoire. Il est possible que ce phénomène ait été induit par le libellé de la question qui demandait de désigner le professeur préféré et non le meilleur. Le fait que la gentillesse soit la qualité largement la plus citée va dans ce sens. Comme dans notre société on trouve normal qu'un professeur soit compétent, c'est une façon de mettre l'accent sur les qualités humaines. Significativement le professeur LOCKHART, notoirement incompétent bien que 
relativement sympathique, n'est jamais cité, ni en bien ni en mal : il est totalement absent des réponses au questionnaire. Cette aptitude à différencier le professeur compétent du professeur sympathique est l'indice d'une certaine maturité des élèves qu'il faudrait croiser avec d'autres indicateurs.

Le jugement porté sur les matières enseignées à Poudlard n'a bien sûr qu'un intérêt limité par lui - même; toutefois, les raisons qui sont invoquées pour le justifier peuvent être analysées. Pour la plupart d'entre elles, elles relèvent de trois catégories: celles qui concernent le professeur, celles qui mettent en jeu l'utilité supposée de la matière et celles relatives à l'intérêt intrinsèque de la discipline. Les trois critères apparaissent en positif pour les disciplines considérées favorablement (professeur sympathique ou compétent, discipline utile, discipline intéressante) et en négatif pour les disciplines peu appréciées (professeur antipathique ou incompétent, discipline inutile, discipline peu intéressante).

Les trois catégories sont de poids à peu près égal. De plus, les justifications concernant les disciplines peu appréciées sont deux fois plus nombreuses que celles concernant les disciplines préférées: il est souvent plus facile de savoir pourquoi on déteste que pourquoi on aime. Enfin, l'impact positif du professeur semble être assez faible alors que son influence négative est beaucoup plus marquée : un bon professeur peut difficilement faire apprécier une discipline dénuée d'intérêt et d'utilité; par contre, un mauvais professeur peut faire détester une matière intéressante et utile. On vérifie ici l'extrême importance du rôle du professeur. Ainsi le cours de préparation de potions magiques du professeur ROGUE est l'enseignement de Poudlard le plus cité. Il ne laisse donc pas indifférent et recueille des opinions favorables en raison de son utilité et son intérêt, et des opinions défavorables majoritairement imputées au caractère antipathique de ROGUE.

Sur ce point, l'enquête préliminaire a fait émerger des indicateurs plus rationnels qui vont permettre de déterminer les raisons pour lesquelles un élève s'identifie à l'enseignement - apprentissage d'une discipline plutôt que d'une autre, en fonction des critères de compétence professionnelle et / ou relationnelle de l'enseignant, mais aussi des critères fondés sur l'intérêt spéculatif ou utilitaire de la discipline.

17 Concernant le parallèle Poudlard / école française, un certain nombre de disciplines enseignées à Poudlard sont considérées comme ressemblant aux matières de nos écoles et collèges : l'histoire de la magie est associée à l'histoire ; le cours concernant les plantes magiques est, selon l'âge, associé aux SVT ou bien à la botanique ; le quidditch est bien sûr associé à l'EPS. Quant au cours concernant les potions magiques, le plus fréquemment cité dans cette réponse, il est le plus souvent associé à la chimie, ce qui n'est pas surprenant. 
Les disciplines dont l'introduction à Poudlard est préconisée sont scientifiques, littéraires, artistiques. La répartition des réponses est homogène et aucune corrélation n'a été notée, excepté avec le niveau scolaire: l'introduction des mathématiques est souhaitée essentiellement par les élèves du primaire alors que l'introduction des disciplines littéraires ou artistiques est préconisée par les sondés les plus âgés. Il est probable que ce phénomène soit dû à l'influence du milieu familial, très importante chez les plus jeunes: les mathématiques sont considérées comme une discipline clé pour l'orientation par de nombreuses familles.

Il n'a été possible de trouver que quelques corrélations entre les réponses car une recherche systématique par des méthodes sans à priori telle l'analyse discriminante n'ont pu être mises en œuvre: elles nécessitent une refonte du questionnaire, en particulier l'introduction d'indicateurs sociologiques et une augmentation de la taille de l'échantillon testé. Ceci constitue le futur développement de ce travail.

\section{La transposition des disciplines dansl'emploi du temps parallèle d'une élève de CE1 lectrice d'Harry POTTER}

Nous avons mené des entretiens semi - directifs avec une lectrice experte d'Harry POTTER sur deux ans (CE1 - CE2), portant sur ses réécritures personnelles de la série. Les productions privées d'autres élèves ont été sollicitées à l'occasion pour décadrer le corpus. Le protocole d'entretien abordait, à parts égales et successivement, le sens et la forme des écrits et le processus d'écriture. Parmi les hypertextes produits, l'emploi du temps parallèle occupe une place centrale car il condense les principaux aspects de la problématique interdidactique. Celle - ci est une pièce - clef de la professionnalité de l'élève que la coexistence des disciplines oblige à gérer des constructions identitaires multiples, tâche pour laquelle il lui faut pouvoir articuler les savoirs disciplinaires autrement que sur le mode utilitaire à court, moyen ou long terme (la note, l'orientation, la profession) mais en ayant accès à la raison épistémique (quand, comment, pourquoi et où un savoir s'est constitué, quels sont ses liens avec les autres savoirs, les autres pratiques sociales).

21 S. L., qui a lancé cette pratique d'écriture dans son groupe d'amis, a tiré son idée d'une allusion du tome 5 à un emploi du temps qui n'est pas décrit ${ }^{4}$. Le rapprochement de l'univers de la fiction et de l'univers de référence a été facilité par le contexte architectural: l'ancien couvent néo - gothique qui abrite l'Institut Universitaire de Formation des Maîtres et quelques classes primaires d'horaires aménagés, dont le CE. L'emploi du temps de la classe a servi de modèle pour placer les horaires et les durées. Les numéros de salles affectés aux différents cours ont été inspirés par les salles environnantes de l'institut universitaire. Les disciplines réelles ont influencé le choix et la disposition des disciplines fictives. 

mentionner les embrayeurs autobiographiques. Pour tous les élèves de ce niveau, lire cinq volumes est une prouesse valorisante. Personnellement, S. L. dit avoir conçu son projet un après - midi de classe où elle luttait contre le sommeil. Des annexes sont venus assez vite compléter l'emploi du temps : devoirs pour chaque soir de la semaine, feuilles d'examen remplies et corrigées, bulletins scolaires, fiche d'orientation faisant état des projets professionnels à long terme, et pendant les vacances d'été, projet d'une école primaire préparatoire à Poudlard.

égime énonciatif est autobiographique, S. L. se présentant comme une élève de Poudlard et s'adressant des billets signés de tel ou tel personnage de la fiction, ou en adressant. La nature des textes autorise à penser que l'on peut étendre les conclusions de PENLOUP 59 sur l'écriture privée des adolescents aux enfants du cycle 3 et même du cycle 2, notamment en ce qui concerne l'écriture de liste, présente dans les « œuvres listes » (comme l'emploi du temps) et les inserts hypertextuels (listes de fournitures scolaires, bons de commande de farces et attrapes, bulletins scolaires, diplômes). Toutefois les listes des 8 ans se font remarquer par leur tendance au mimétisme, tendance dont on ne peut dire sans enquête supplémentaire si elle est due à l'âge ou au contexte de la réécriture observée. Ils reproduisent ou empruntent les supports sociaux : feuilles d'agendas scolaires pour l'emploi du temps, feuilles noircies imitant le parchemin pour les diplômes, bordereaux pour les commandes.

L'analyse du codage métaphorique de l'emploi du temps montre au départ un comportement analogique, fiction et réalité étant rapprochées aussi bien sur la base $d u$ signifiant («mathématiques » / «métamorphoses ») que sur celle du signifié. Mais dans ce dernier cas le rapprochement a toujours une base autobiographique : le cours jugé le plus important dans l'école réelle et qui correspond à la lutte contre les forces du mal est, selon les élèves, le cours préféré ou le cours redouté. L'invention analogique est ensuite développée par un

descriptif réaliste prenant en compte les contenus, les activités et l'importance hiérarchique des disciplines. Le cours de quidditch remplace le sport ou la danse. Le cours de potions magiques, la botanique et la zoologie magiques sont positionnés en face des sciences, avec quelques hésitations dues à l'indifférenciation formelle de ces matières dans les programmes de l'école primaire française. Implicite au moment de l'écriture, la hiérarchisation, est facilement explicitée durant l'entretien. L'aller - retour entre fiction et réalité active les mécanismes de symbolisation: ainsi S. L. estime que si elle recommençait, elle choisirait de mettre en correspondance avec les forces du mal, non pas le cours de conjugaison, mais le français dans son ensemble, parce que «quand sait discuter et écrire, on peut lutter contre les forces du mal ». Une troisième étape est franchie lorsque les disciplines hiérarchisées sont perçues en diachronie dans l'histoire générale et dans la progression curriculaire. Dans l'école primaire des sorciers, l'alchimie devient alors la propédeutique du cours de potions. 


\section{Pré - expérimentations}

Deux (pré-) expérimentations ont été menées en février 2004, portant sur les rapports que les élèves sont susceptibles d'établir entre une fiction littéraire à intertexte scientifique et leurs connaissances scientifiques.

La première expérimentation (sciences de la terre) a été menée avec une classe de 24 élèves d'un cours double CM1 - CM2. Il a d'abord été demandé aux élèves une lecture silencieuse d'un extrait du tome 1 d'Harry POTTER (la visite à la banque souterraine des Gringotts), puis de remplir un questionnaire individuel relatif au texte lu. Voici quelques unes des questions :

- Dessine le monde sous nos pieds (« d'après ce que tu as lu dans le texte »et « d'après ce que tu connais »).

- A quoi ressemble le passage qui mène à Gringotts, la banque des sorciers située à des kilomètres en sous-sol?

- Existe - $\mathrm{t}$ - il sur la Terre des passages qui permettent de se retrouver dans un endroit différent de celui d'où l'on vient?

- Voici des groupes de mots ou des phrases qui se trouvent dans les deux derniers paragraphes, range-les dans le tableau ci - dessous (Ce qui peut être vrai - Ce qui n'est pas vrai Je ne sais pas) a - un vent glacial b - un dragon c - Ils longèrent un lac souterrain bordé de stalactites et stalagmites. $\mathrm{d}$ - En s'enfonçant encore davantage dans les entrailles de Gringotts, la température devenait de plus en plus glaciale.

Seul un enfant a produit un dessin proche de ce qui était raconté dans le texte. 18 élèves ont produit un dessin qui ressemblait très fortement aux images du film et n'ont pas tenu compte des indices textuels. La notion de sous - sol semble très confuse pour ces enfants qui ont eu du mal à imaginer un monde souterrain qui ne soit pas un monde magique. Enfin, malgré le travail réalisé lors d'une étude préalable des volcans, avec la mise en évidence d'une augmentation de la température en fonction de la profondeur, seuls 3 enfants (dont 1 n'avait pas suivi les activités sur le volcanisme) ont produit un énoncé («plus on s'enfonce, plus c'est chaud ») en accord avec la loi établie scientifiquement. La seconde expérimentation a été menée avec une classe de CM2 en sciences de la vie (botanique). Les enfants devaient lire un extrait qui décrit une scène de d'apprentissage chez les jeunes sorciers concernant la mandragore. Cette plante, support de nombreuses légendes en raison de l'aspect parfois anthropomorphique de sa racine, est pourtant réelle. Mandragora Officinalis appartient à la famille des solanacées comme la belladone. Les élèves devaient classer les différentes phrases ou morceaux de phrases de l'extrait dans un tableau à deux colonnes ("Vérité scientifique ", "Fiction»). L'enseignante a proposé ensuite une lecture d'une définition de la mandragore tirée d'un dictionnaire encyclopédique et a demandé aux élèves de réviser leur première classification. Ils ont alors énuméré leurs erreurs et constaté leurs défaillances. 
en ressort que les élèves ont montré un vif intérêt pour la mandragore, mais confondent vérités scientifiques et faits réalisables dans la fiction. Ceci peut être dû à une particularité de Harry POTTER où la différence entre les univers réel et fictionnel est reprise métaphoriquement au sein de la fiction même sous forme de la différence entre l'univers des moldus et celui des sorciers. Or cette métaphore entraine dans le roman une autre figure qui est la métalepse, « cette figure par laquelle un écrivain [...] se représente comme produisant lui - même ce qu'il ne fait, au fond, que raconter ou décrire $»^{6}$. La reprise en miroir de la partition réel/fiction de notre univers par l'opposition monde des moldus/monde des sorciers a des effets contradictoires. D'un côté, elle peut aider à surmonter l'illusion, puisqu'elle rappelle le statut fictionnel de la fiction ; mais de l'autre, elle conforte cette même illusion, en donnant l'impression que notre propre vision du monde est le produit et non pas la source de l'univers des moldus et des sorciers, ce qui, pour certains enfants peut contribuer à augmenter la confusion entre fiction et réalité au lieu de la dissiper.

En outre, choisie par ROWLING pour sa position historique entre une proto - science (la médecine par les plantes) et une vraie science, la botanique, la mandragore ajoutait sa propre métalepse. C'est une plante du monde réel, objet de la botanique "réelle », mais qui est donnée comme une plante magique dont les pouvoirs ne se manifestent qu'aux sorciers, personnages de la fiction. Les réponses au questionnaire ne pouvaient qu'être perturbées par ce statut métaleptique propre aux objets et aux créatures de la littérature fantastique. Il aurait peut - être fallu commencer par travailler sur une plante appartenant sans conteste au seul univers fictionnel, par exemple le Mimbulus mimbletonia de Neville LONDUBAT (apparue dans le Tome 5, l'ordre du Phénix). Cela étant, à la question: "Dans quelle discipline avez-vous travaillé aujourd'hui?»; les élèves répondent en majorité : «en sciences!». Ceci tend à prouver que pour ces élèves, la définition de la science ne dépend pas du caractère « réel » des objets - heureusement ! L'accès au véritable statut de l'objet scientifique : réel mais abstrait en sera d'autant facilité ! - mais aussi des opérations cognitives et discursives et des objectifs : observer, classer, expliquer.

31 Ces deux expérimentations nous amènent à penser qu'une lecture, même attentive, d'un extrait de quelques dizaines de lignes d'une œuvre telle que Harry POTTER ne semble pas pouvoir permettre au lecteur de débrouiller ce qui ressortit à l'univers « réel » (la « Vérité scientifique ») de ce qui ressortit à l'univers fictionnel. La taille entre aussi en ligne de compte: la mise en place de l'univers fictionnel (d'autant plus qu'il est éloigné de l'univers quotidien) se fait tout au long de l'œuvre par une série de procédés qui doivent fonctionner sans être visibles (ECO : Apostille au Nom de la rose). Pour les démonter, un extrait est toujours trop court.

\section{Lector in speculo fabulae : le « miroir du Riséd » comme dispositif interdidactique}

Dans ce roman scolaire, les disciplines convoquées par l'auteur sont inégalement représentées si bien que les arts plastiques, l'éducation musicale et l'éducation physique 
et sportive ne sont pas explicitement enseignés à l'école des sorciers. Il est donc intéressant d'opérer une lecture ciblée dans la perspective de la didactique des Arts Visuels pour vérifier si les arts n'étaient pas implicitement évoqués par l'auteur.

L'analyse des quatre premiers tomes du roman et des trois films réalisés à ce jour, montre que les arts visuels et l'histoire des arts sont fortement sollicités par J.K.ROWLING. Déjà elle fait référence à des œuvres précises: le château de Neuschwanstein ${ }^{7}$ évoqué par l'architecture de Poudlard, "la dame à la licorne $"^{8}$ ou encore les lithographies de M.C ESCHER, réalisées entre 1951 et 1959, dont les escaliers ouvrent sur des espaces paradoxaux qui défient nos modes habituels de représentation.

Mais le plus remarquable demeure les dispositifs fictionnels décrits dans le roman: les "Chocogrenouilles " ${ }^{9}$; les "Beuglantes"; le journal interactif de Tom JEDUSOR ${ }^{10}$ et le " miroir du Riséd ${ }^{11}$ (psyché magique montrant les désirs du sujet qui s'y regarde). L'interprétation par les arts visuels et le multimédia semble littéralement programmée par le roman, dont les descriptions sont assez précises pour être transposées directement. L'idée nous est alors venue de détourner une de ces mises en abyme pour permettre à des lecteurs de mettre en scène leur lecture et d'y être ensuite confrontés par vidéo observation. Nous pourrions ainsi avoir un accès «naturel» aux représentations disciplinaires sur la base desquelles les lecteurs décodent la fiction, et les faire ensuite commenter et travailler dans la perspective d'une meilleure identification des disciplines.

Nous avons décidé de recréer le "miroir du Riséd », en deux phases successives. D’abord, notre dispositif devait permettre aux sujets - lecteurs (adultes et enfants) de vivre leurs fantasmes de lecture privée en usant de leur capacité de projection et d'interidentification $\mathrm{au}(\mathrm{x})$ personnage(s) de la fiction. Par la suite, ce même dispositif devait les amener à expliciter leur perception des disciplines enseignées dans le réel et à l'école des sorciers.

Le Riséd, emprunté par ROWLING à l'imaginaire du conte merveilleux reflète les désirs de celui qui s'y regarde sous la forme d'une image intemporelle et fantasmée.

La structure narcissique du moi tient un rôle important dans l'économie libidinale et dans la construction de la personnalité de l'enfant. L'une des hypothèses de cette première phase de l'expérimentation était qu'à la manière du conte merveilleux mais à travers une installation multimédia personnalisée, le lecteur pourrait grâce au jeu (JE) exprimer implicitement les angoisses, les craintes mais aussi les désirs liés à sa croissance. Sur ce point, Bruno BETTELHEIM souligne, « (...) qu'en brodant des rêves éveillés, en élaborant et en ruminant des fantasmes issus de certains éléments du conte qui correspondent aux pressions de son inconscient (...) l'enfant transforme ces contenus pour mieux y faire face $»^{12}$.

En raison de ressemblances architecturales, évoquées par nos lecteurs experts, entre Poudlard et l'IUFM George V à Nice, nous avons choisi une cave voûtée, située dans les sous - sols du centre niçois, comme lieu de nos installations. Comme acteurs, nous avons pressenti quatre sujets âgés de 9 ans et demi à 39 ans qui avaient tous répondu au 
questionnaire sur la lecture. Lors d'un entretien semi - directif filmé, nous les avons invités à formuler une proposition divergente d'un état de la fabula ${ }^{13}$ existante, en leur demandant d'imaginer ce que le «miroir du Riséd » renverrait comme projection des désirs pour leur personnage préféré. Ils ont imaginé une séquence fictionnelle à partir d'une image infographique de base, choisie parmi douze propositions, mettant en scène un miroir dans un lieu (intérieur ou extérieur). Grâce aux arts plastiques et aux techniques du multimédia, nous avons pu matérialiser leur échappée hors du texte initial en créant une transposition audiovisuelle, sorte d'hypertexte ${ }^{14}$ au carré (écriture de la lecture) du texte de ROWLING.

Techniquement, chaque installation contractualisée a été conçue comme la vidéo projection d'une série d'images numériques de grandes dimensions accompagnée d'une bande son. Un rituel d'immersion devait aider le sujet à prendre confiance en ses capacités et à se motiver (reprenant le concept du « Patronum » défini par l'auteur dans le troisième tome du roman). Pour chacun, c'est le comportement oral et gestuel qui a déterminé le rythme de succession des images et l'interactivité qui en découlait. De ce fait, la durée de chaque installation a été aléatoire, sans dépasser toutefois une dizaine de minutes, la «magie » du dispositif étant essentiellement liée à l'utilisation d'images numériques, immatérielles comme un rêve.

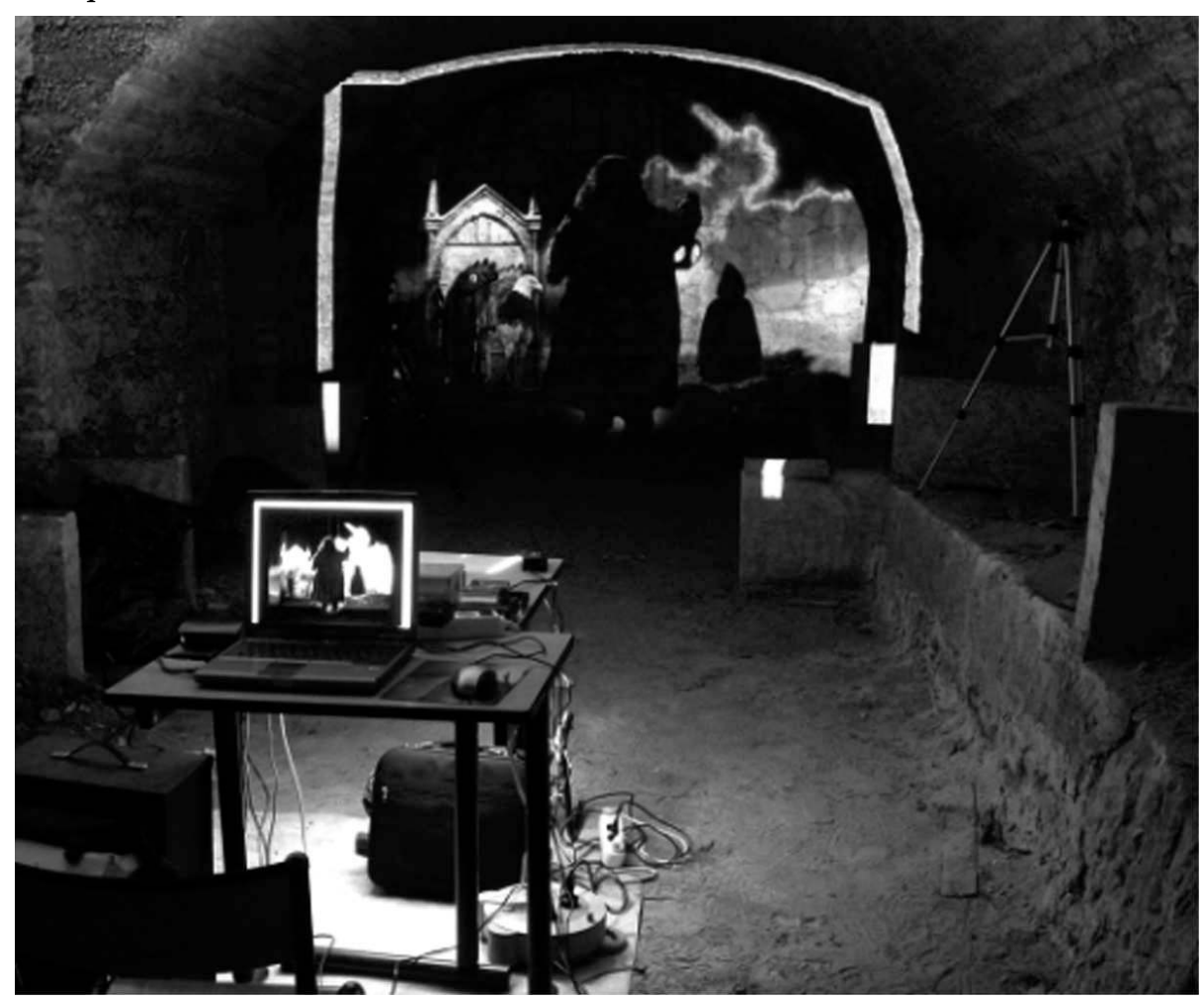

Tirant partie des compétences de lecture experte des lecteurs, ce dispositif a permis les va-et-vient entre situations vécues et situations fictionnelles. Les sujets - lecteurs se sont fortement impliqués comme en témoignent les photographies et les films. Quelques minutes en général ont suffi pour qu'ils oublient la présence de la caméra et le regard de l'autre, devenant réellement les acteurs de leur propre fantasme de lecture. Aussi ces installations ne sont-elles pas de simples représentations du fantasme, elles en dévoilent la phénoménologie grâce à la coopération du lecteur - spectateur - acteur, du plasticien et des images interactives. La deuxième partie de notre projet commence à présent. L'analyse de la motivation des personnages de la fiction retenus par nos lecteurs devrait 
nous renseigner sur leurs propres conduites scolaires. En effet, comme le soulignent Pierre GLAUDES et Yves REUTER dans leur ouvrage consacré au personnage, celui - ci contribue à l'inscription des valeurs dans le récit, c'est à dire à son axiologisation.

La plupart des personnages du roman Harry Potter sont soit des élèves ou des professeurs soit des membres d'une communauté éducative. Leur appartenance à l'une des quatre «maisons» (Gryffondor, Poufsouffle, Serdaigle et Serpentard) reflète leurs prédispositions naturelles à certains apprentissages et à certaines conduites de projets, tandis que leurs comportements scolaires montrent comment ces prédispositions sont modifiées par le caractère et surtout les images de soi que chaque discipline renvoie à l'apprenant. Sur cette base, l'identification à l'un des personnages de la fiction trouve son prolongement dans les «propositions fictionnelles disciplinaires» que chaque lecteur fera, et qui permettra de faire émerger son vécu scolaire à la faveur de la comparaison entre les disciplines fictives et les disciplines réelles. Au préalable, une enquête et de nouveaux questionnaires seront réalisés pour préciser ce qui, aux yeux des lecteurs, rend emblématique et reconnaissable chaque discipline. On espère ainsi mieux connaître l'incidence du rapport entre l'image que les élèves ont des disciplines et leur image de soi sur le processus d'identification qui est le préalable à tout apprentissage.

\section{Conclusion}

Une première remarque concernera la mise en rapport des corpus fictionnels et des situations d'apprentissage. Indubitablement l'œuvre complète fournit un socle d'expertise commun fort, que ne remplace pas dans l'approche de l'extrait la culture commune de la transposition cinématographique. Les lecteurs les plus experts sont aussi ceux qui sont les plus entraînés à l'abstraction définitionnelle et comparative, les rescripteurs manifestant en outre de meilleures compétences justificatives et explicatives.

La lecture experte de la série prédispose à la classification et à la systématisation, tandis que la lecture d'extraits, plus facile à instrumenter, se prête davantage à la comparaison fine des descriptions et dialogues du roman avec les pratiques et discours disciplinaires réels. Facilitant le travail sur les situations disciplinaires (fantasmées, réécrites, confrontées par vidéo - observations), elle prédispose à l'action réfléchie, à deux conditions :

- renforcer au préalable les activités disciplinaires du français sur le dévoilement de la fabrique du texte, sous peine de voir les élèves rester prisonniers de l'effet de réel ;

- faire porter le questionnement sur l'analyse précise des discours scientifiques et littéraires en prenant des points de comparaison dans les écrits des disciplines évoquées par la fiction.

Une deuxième remarque portera sur l'interaction entre l'activité de réécriture et les apprentissages disciplinaires. Obligeant à retravailler les systèmes axiologiques des personnages, la réécriture fictionnelle provoque une montée en lucidité des acteurs scolaires sur leurs stratégies et leurs motivations. Evaluer la précision des descriptions ouvre des perspectives sur les contenus et objectifs disciplinaires, ainsi décadrés de leur 
usage habituel, ceci bien entendu à partir du moment où la fonction de la littérature comme représentation et interrogation de la réalité a été reconnue et intégrée.

Notre troisième remarque mettra en valeur la différenciation des pratiques sociales comme condition aussi bien que comme conséquence de la différenciation des disciplines. La séparation des entrées lecture - écriture privée / lecture - écriture scolaire a permis de saisir les points de passage, les points de clivage et les croisements entre images de soi et images des disciplines liées aux deux domaines. On ne peut qu'être frappé par l'image du scolairement correct qui ressort des représentations du discours privé des élèves, et même des très bons élèves : une association de rébarbatif, d'opaque et d'arbitraire, qui reflète la conception du grand public (le français sans dictée n'est pas du français, les mathématiques sans tables de multiplication apprises par cœur ne sont pas des mathématiques). Pourtant mieux vaut accueillir les stéréotypes disciplinaires que les ignorer. En effet, leur persistance est un bon indicateur des repères dont les novices ont besoin pour se rassurer, et des endroits où devra porter le travail de problématisation et d'intégration.

Nous terminerons en insistant sur l'importance du background cosmogonique des apprentissages disciplinaires. La vision du monde est l'englobant social des disciplines. Une des raisons du succès de la série vient de ce qu'il n'y a pas rupture mais continuité entre le monde des moldus (c'est - à - dire du réalisme scientifique technique) et celui des sorciers. Il est épistémologiquement exact et psychologiquement rassurant d'admettre, si on les différencie, l'égale dignité des points de vue magique, scientifique mais aussi totémique, ou analogique, puisque, de toute façon, ils coexistent en chaque sujet.

\section{BIBLIOGRAPHIE}

BECKETT L. S., (2001), « Livres pour tous : le flou des frontières entre fiction pour enfants et fiction pour adultes ", Tangence, «L'écriture pour la jeunesse : de la production à la réception », $\mathrm{n}$ ${ }^{\circ} 67$ pp. 10 - 1.

Bettelheim B. (1976), Psychanalyse des contes de fées. Paris : Editions Robert Laffont.

DE SINGLY F. (1992), L'enquête et ses méthodes : le questionnaire. Paris : Nathan.

ECO U., (1985.), Lector in fabula ou La coopération interprétative dans les textes narratifs. Paris : Editions Grasset.

LACAN, J., (1949), « Le stade du miroir comme formateur de la fonction du Je, telle qu'elle nous est révélée dans l'expérience psychanalytique » communication faite au XVIe Congrès international de psychanalyse à Zürich. Première version parue dans la Revue Française de Psychanalyse, volume 13, n4, pp $449-455$.

MARC E. (2004), « La construction identitaire de l'individu », Identité(s), l'individu, le groupe, la société, Sciences humaines, p. 37.

PENLOUP M - Cl., (1999) L'écriture extra - scolaire des collégiens, Des constats aux perspectives didactiques. ESF. 
REUTER Y. (2003), L'analyse du récit. Paris : Nathan. « Collection Littérature 128 ».

REUTER Y. \& GLAUDES P. (1998), Le personnage. Paris : PUF. « Que sais - je ? 3290.

ROWLING J.K., (2003), L'ordre du phénix, trad. Française. Paris : Gallimard.

ROWLING J.K., (1999), Harry POTTER à l'école des sorciers, traduit de l'anglais par Jean - François MENARD. Paris : Gallimard.

ROWLING J.K., (1999), Harry POTTER et la chambre des secrets, traduit de l'anglais par Jean François MENARD. Paris : Gallimard.

WATZLAWICK P., (1981), Changements, paradoxes et psychothérapie. Paris : Seuil.

\section{NOTES}

1. Cf. WATZLAWICK, (1981).

2. Cf. MARC (2004) p. 37: "les identifications ne procèdent pas seulement des groupes d'appartenance mais aussi des groupes de référence dans lesquels le sujet puise ses modèles ou auxquels il cherche à s'intégrer ». Chaque matière enseignée relie l'élève en même temps à un groupe d'appartenance, le groupe - classe et à un groupe social de référence : celui de la discipline.

3. Comme le laissaient supposer déjà les études réalisées sur le lectorat de la série, cf. BECKETT (2001), pp. 10 - 12.

4. ROWLING, J.K., L'ordre du phénix p. 257 : «Regarde. Ce qu'on a aujourd'hui, dit Ron d'un ton grincheux en mettant son emploi du temps sous le nez de Fred. C'est le pire lundi que j'aie jamais vu. »

5. PENLOUP (1999).

6. FONTANIER (1977), Les figures du discours, Flammarion, p. 128.

7. Le château de Neuschwanstein a été construit entre 1869 et 1881 . Son style médiéval et wagnérien peut être rattaché au néo - gothique (1840 - 1880) et au néo - roman (1860 - 1900).

8. "La dame à la licorne ", vers 1500 . Série de six tentures murales tissées et réalisées à Bruxelles rappelant le style du peintre Hans MEMLING. Les tentures illustrent les cinq sens. Celle appelée «mon seul désir » illustrerait le sixième sens, celui du cœur et de l'entendement. Elles sont actuellement exposées au Musée National du Moyen - ge, Hôtel de Cluny à Paris. J.K ROWLING utilise cette œuvre pour décorer les murs de la salle commune de la maison Gryffondor. Voir Chris COLUMBUS, Harry POTTER à l'école des sorciers, Warner Bros Vidéo 2002 - Chapitre 13 du DVD et, Harry Potter et la chambre des secrets, Warner Bros Vidéo 2003 - Chapitre 24 du DVD.

9. ROWLING, J.K., (1999), Harry POTTER à l'école des sorciers, pp. 105 - 107.

10. ROWLING, J.K., (1999), Harry POTTER et la chambre des secrets, pp. 254 - 263.

11. ROWLING, J.K., (1999), Harry POTTER à l'école des sorciers, pp. 206 - 208.

12. BETTELHEIM (1976) p. 17 "Pour pouvoir régler les problèmes psychologiques de la croissance (déceptions narcissiques, dilemmes oedipiens, rivalités fraternelles, affirmer sa personnalité, prendre conscience de sa propre valeur et de ses obligations morales) l'enfant a besoin de comprendre ce qui se passe dans son être conscient. (...)».

13. ECO (1985). p. 132 « (...) La fabula est le schéma fondamental de la narration, la logique des actions et la syntaxe des personnages, le cours des évènements ordonnés temporellement. Elle peut ne pas être une séquence d'actions humaines et porter sur une série d'évènements qui concernent des objets inanimés ou même des idées ».

14. REUTER, Yves dans son ouvrage, L'analyse du récit, définit l'hypertextualité en ces termes: «l'hypertextualité est la relation qui unit un texte B à un texte A qui lui est antérieur et avec 
lequel il ne se situe pas un rapport commentatif mais d'imitation ou de transformation, à des fins ludiques, satiriques ou sérieuses ». Paris: Editions Nathan, 2003. "collection Littérature 128 ». p. 113.

\section{RÉSUMÉS}

Dans la situation de confusion identitaire qui résulte de la coexistence « sauvage » des disciplines enseignées dans l'école, le roman scolaire développé par la série Harry POTTER permet d'apporter une solution systémique en prescrivant le symptôme de confusion. La lecture désacralisante retrouve les réalités disciplinaires que l'auteur et/ou les lecteurs ont projetées sur la fiction. La comparaison des passages qui contiennent des intertextes de chaque discipline avec leurs textes-sources et les diverses réécritures qu'elle autorise favorisent à la fois l'approfondissement intradidactique et l'articulation interdidactique. On trouvera ici des comptes rendus d'expériences menées sur la reconnaissance et l'appropriation de discours disciplinaires en sciences, lettres, histoire, mathématiques, et éducation physique.

The school system faces a situation of identity confusion resulting from the coexistence of various subject matters. A systematic answer known as the prescription of the symptom, applied to the confusion within the school system, is provided by the Harry POTTER saga. When reading this saga in a desacralizing way, one discovers the subject matters realities projected on the fiction by both the author and the readers. Discourses of each subject matter are to be found in various fragments of the novel. And comparing them to their original through rewriting helps going deeper in the approach both of intra didactics and inter didactics. The following accounts of experiments about various recognition and appropriation of subject matters discourses in Sciences, Literature, History, Mathematics, Physical training and Art.

\section{INDEX}

Keywords : anthropological approach, interdisciplinary school learning, professional experience, transmission, type of activity

Mots-clés : enquête, entretien, identité disciplinaire, interdidactique, roman scolaire, vidéo observation

\section{AUTEURS}

\section{NICOLE BIAGIOLI}

Professeur des Universités, Directrice équipe I 3D, IUFM Nice

\section{SOLANGE CARTAUT}

Professeur agrégée d'arts plastiques et formatrice TICE 


\section{JEAN-PHILIPPE DROUHARD}

Maître de conférences en mathématiques, IUFM Nice

\section{JEAN-JACQUES LEGENDRE}

Professeur des Universités en chimie, IUFM Nice 